\title{
The Recruitment and Description of University Students who Self-Report Difficulty Acquiring Early Reading Skills
}

\author{
Melissa McGonnell ${ }^{1}$, Rauno Parrila ${ }^{2}$, \& S. Hélène Deacon ${ }^{1}$ \\ ${ }^{1}$ Dalhousie University, ${ }^{2}$ University of Alberta
}

\begin{abstract}
Self-report is a time- and cost-efficient screening measure that has the potential to be useful as a stand-alone means of recruiting adults for participation in reading research. We report on a sample of university students $(N=46)$ recruited using the Adult Reading History Questionnaire - Revised, half of whom reported early difficulty with reading acquisition. As a group, those reporting early difficulty with reading had poorer current reading skills than those who did not. Some $(n=13)$ of those who reported early reading difficulty appear to have compensated for this. We conclude that self-report has the potential to be used as an effective screening measure in research targeting adults with a history of early reading difficulties.
\end{abstract}

To date, a great deal of reading research has focused on children and it is only recently that adult populations have begun to receive more attention. One particularly interesting adult population is those who have compensated for their earlier reading difficulties. Lefly and Pennington (1991) estimated that 22 to $25 \%$ of children with dyslexia (a specific reading difficulty) will improve their reading to the point that their skills will be well within the normal range by the time they are adults. Research with compensated adults could provide valuable insight into how they accomplish this feat and help optimize remedial interventions for children. While such research is vital, studies with adults who have had early reading difficulties face a number of challenges. Perhaps the foremost of these is the identification of potential participants. 
One possible place to find adults who have compensated for early reading difficulty is in a population of university students. Reading demands for most university classes are high and it seems logical to expect that university students who experienced early difficulty with reading will have developed some compensatory mechanisms. In fact, many researchers have recruited college and university students as participants in reading research on dyslexia (e.g., Bruck, 1990, 1992; Deacon, Parrila, \& Kirby, 2006; Erskine \& Seymour, 2005; Leong, 1999; Miller-Shaul, 2005). Often, these studies have required assessment and/or documentation of participant reading difficulties, either in childhood or while at college or university (Bruck, 1990, 1992; Erskine \& Seymour, 2005, Leong, 1999; Miller-Shaul, 2005).

Requiring previous or current diagnostic information does ensure the selection of participants with reading difficulty; however, limiting participation to those whose difficulties have been documented may reduce the ecological validity of the sample. For example, not every child with reading difficulty has received a psychological assessment. As well, limited time and financial considerations together with privacy issues often mean that researchers cannot access childhood records of adult participants or locate adults for whom childhood records exist. Limiting participation to those adults who have a current diagnosis presents other challenges to the sample's validity. College and university students with documented difficulties typically have this documentation because they have sought accommodations or assistance through student services at their institution. Therefore, they may represent a population that has not compensated as well for early difficulty or that is more aware of their own challenges in comparison to those who have not sought assistance or accommodations from special services. Clearly, both the presence of diagnosable difficulties and the self-selection process involved in recruitment may affect results.

Another method of identifying adults with reading acquisition difficulties is through retrospective self-report. Studies of adult dyslexics who have a documented history of childhood dyslexia have often incorporated self-report as an additional means of screening or of gaining further information about participants (e.g., Erskine \& Seymour, 2005; Fink, 1998). Schulte-Körne, Deimel, \& Remschmidt (1997) found that adults' retrospective reporting of spelling and reading difficulty accurately predicted whether they did or did not have current spelling difficulties (see also Decker, Vogler, \& Defries, 1989). It seems then that retrospective self-report has the potential to be a useful device for screening adult participants and some researchers have indeed used self-report along with other methods (e.g., referral to student services) to screen adult participants (e.g., Deacon et al., 2006); however, to our knowledge, no study 
has examined the effectiveness of self-report as a stand-alone method of screening adult participants for a history of difficulty with the acquisition of reading skills. As a stand-alone measure, self-report would be more time- and cost-efficient to implement and it has the potential to result in a sample that is more broadly representative, especially of individuals who have managed to compensate for their early difficulties.

Adults screened using self-report of a history of reading difficulty are likely to have diverse reading skills. Across a range of screening methods, a host of studies has demonstrated that most adults who had difficulty learning to read continue to experience difficulty with some aspect of reading including fluency (i.e., reading speed), word recognition, phonological awareness (Birch \& Chase, 2004; Bruck, 1990, 1992; Deacon, et al., 2006; Leong, 1999; Miller-Shaul, 2005; Wilson \& Lesaux, 2001), and morphological processing (Deacon et al., 2006). However, if Lefly and Pennington's (1991) estimate that approximately one-quarter of children with early reading difficulties compensate for these by adulthood is accurate, it is reasonable to expect that not all adults with a history of reading difficulty will show the same degree of impairment in their current reading skills.

We know little about how variable the reading skills of university students who self-report early reading difficulty might be because this population has yet to be characterized in terms of their reading skills. The primary goal of this study was to do this. To this end, we addressed three specific questions. First, do university students who report reading acquisition difficulties in childhood demonstrate, as a group, less well developed reading skills than students who do not report early difficulty? Second, do all individuals who report reading acquisition difficulties in childhood continue to show at least some signs of difficulty so that their profiles of reading ability are distinguishable from those who do not report any early difficulty? Third, what proportion of university students who report early difficulty with the acquisition of reading skills also report receiving formal diagnoses of reading disability/dyslexia in childhood or as adults? Answering these questions will allow us to examine the validity of self-report as a means of selecting individuals with a history of reading difficulties. 
McGonnell, Parrila \& Deacon

\section{Method}

\section{Participants}

Participants in this study were selected based on their responses on a screening measure, the Adult Reading History Questionnaire - Revised (ARHQ-R; Parrila, Corkett, Kirby, \& Hein, 2003). While this measure asks students to provide information about a number of parameters of their reading history in a total of 67 questions, only information about their experiences with reading in elementary school (nine questions) was used to determine their eligibility for the study. (See Parrila et al., this issue, for a more detailed description of the questionnaire and the questions.) Elementary school was chosen because we were interested in the ability to compensate for early reading difficulties. Participants were asked to rate their attitude or experience about the following: having difficulty learning to read, requiring extra help to learn to read, reversing the order of letters, having trouble learning letter or colour names, comparing reading skill to that of other students in elementary school, personal attitude to reading in school, amount of reading for pleasure, comparing reading speed to that of other students in elementary school, and difficulty learning to spell.

The ARHQ-R was administered to 777 participants as part of a broad eligibility screening of students taking an introductory psychology course and 702 of these students agreed that their data could be used for secondary research (other than screening for participation in research studies). The 702 participants' scores from the nine questions about elementary school were summed and divided by the highest possible score to calculate a proportion score for each participant. Scores could range from a low of 0 to a high of 1, with lower scores indicating less difficulty with reading in elementary school. Of the 702 participants, 450 scored between 0 and 0.25 and were eligible for inclusion in the No Reported Reading Difficulty (NRD) group, while 167 received scores greater than 0.37 and were eligible for inclusion in the Reported Reading Difficulty (RRD) group. These criteria were based on previous research (e.g., Deacon et al., 2006; Lefly \& Pennington, 2000). All 617 eligible participants were invited to volunteer for participation in this study and were contacted individually once they did so. This procedure resulted in an initial sample of 23 students in each group; however, the data from four students (two for whom English was a second language, one who reported sustaining a head injury, and one for whom there was experimenter error) were not included in the analyses. This left 21 participants in each group (NRD: $M=.10, S D=.08$; RRD: $M=.54, S D=.15$ ). 


\section{Measures of Reading Skills}

All participants completed a battery of tests designed to assess various reading skills. The reading tests were the Word Identification subtest from the Woodcock Reading Mastery Tests-Revised (Woodcock, 1987) and the Comprehension subtest from the Nelson-Denny Reading Test (Brown, Fishco, \& Hanna, 1993). The Nelson-Denny test consists of two indices: reading rate (calculated at one minute) and reading comprehension (with a 20 minute time limit). To obtain an estimate of untimed reading comprehension ability, the number of correctly answered comprehension questions was divided by the number of attempted questions to calculate an overall percentage of correct questions (as in Deacon et al., 2006). The phonological processing test was the Blending Non-words subtest from the Comprehensive Test of Phonological Processing (CTOPP; Wagner, Torgesen, \& Rashotte, 1999). All tests were normed on populations whose age and/or education level were similar to the participants in this study and the reliabilities reported in the manuals for each subtest (specific to the adult or college normative sample) were moderate to high (Word Identification, Spearman-Brown split-half reliability coefficient $=$ .97; Reading Rate alternate forms $r=.68$; Reading Comprehension alternate forms $r=.81$; Non-word Blending $\alpha=.81$ ).

\section{Results}

\section{Comparison of Current Reading Skills}

The first question examined was whether university students who reported early difficulty with reading acquisition (Reported Reading Difficulty; RRD group) currently demonstrated less well developed reading skills than those students who did not report early difficulty with reading acquisition (No Reported Reading Difficulty; NRD group). A multivariate analysis of variance (MANOVA) was computed to examine group differences in five reading skills (word identification, reading rate, timed reading comprehension, untimed reading comprehension, and phonological awareness). Overall differences were found between the groups, $F(5,36)=6.463, p<.001, \eta^{2}=.437$. Univariate results are reported in Table 1. The NRD group had better word identification skills and better timed and untimed reading comprehension skills than the RRD group. There was a trend towards significance for group differences on phonological awareness and reading rate. 
McGonnell, Parrila \& Deacon

Table 1

Means and Partial Eta Squared of Performance of NRD and RRD Groups on Reading and Phonological Processing Tests. F-values Report the Main Effect of Group

\begin{tabular}{|c|c|c|c|c|}
\hline & \multicolumn{2}{|c|}{ Group } & & \\
\hline & $\begin{array}{c}\text { NRD } \\
(n=21)\end{array}$ & $\begin{array}{c}\text { RRD } \\
(n=21)\end{array}$ & & \\
\hline Tests & $\begin{array}{c}M \\
(S D)\end{array}$ & $\begin{array}{c}M \\
(S D)\end{array}$ & $F$ & $\eta^{2}$ \\
\hline \multicolumn{5}{|l|}{ Reading } \\
\hline WRMT Word Identification (RS) & $\begin{array}{l}99.81 \\
(3.08)\end{array}$ & $\begin{array}{l}93.57 \\
(4.70)\end{array}$ & $25.93^{* *}$ & .393 \\
\hline ND Rate (RS) & $\begin{array}{l}208.48 \\
(39.00)\end{array}$ & $\begin{array}{l}186.71 \\
(51.25)\end{array}$ & 2.40 & .057 \\
\hline ND Timed Comprehension (RS) & $\begin{array}{l}57.71 \\
(9.93)\end{array}$ & $\begin{array}{l}44.19 \\
(9.38)\end{array}$ & $20.60 * *$ & .340 \\
\hline ND Untimed Comprehension (\%) & $\begin{array}{l}87.33 \\
(9.96)\end{array}$ & $\begin{array}{c}78.76 \\
(11.77)\end{array}$ & $6.49 *$ & .229 \\
\hline \multicolumn{5}{|l|}{ Phonological Processing } \\
\hline CTOPP Non-Word Blending (RS) & $\begin{array}{l}10.76 \\
(2.47)\end{array}$ & $\begin{array}{c}9.43 \\
(2.60)\end{array}$ & 2.91 & .068 \\
\hline
\end{tabular}

Note . WRMT $=$ Woodcock Reading Mastery Tests; $\mathrm{ND}=$ Nelson-Denny $;$ CTOPP $=$ Comprehensive Test of Phonological Processing; RS = Raw Score.

$* p<.05 ; * * p<.001$

\section{Individual Differences in Reading Skills}

We were also interested in whether all university students who reported difficulty with reading acquisition would continue to demonstrate detectible difficulties with reading skills. This question was more complex to answer. First, we characterized the reading skills of participants in both groups. Next, 
we examined the reading scores of participants in the RRD group to allow a determination of which participants had and had not compensated for early reported reading difficulty. Finally, we determined whether the reading skills of individuals who had compensated for early reported reading difficulty could be distinguished from the reading skills of those who reported no early difficulty with reading acquisition.

For the purposes of addressing this question, difficulty was defined as scoring more than one standard deviation below average. Raw scores were converted to standard scores, percentiles, or z-scores depending on the measure. For the first four measures discussed (word identification, phonological processing, reading rate, and timed reading comprehension), average was defined according to the standardization sample used in the norming of the test. For untimed reading comprehension, a standardization sample was not available. We elected to use the scores for the NRD group as a comparison benchmark and so average was defined as the mean of this group. The mean and standard deviation of the NRD group was then used to calculate z-scores for participants in the RRD group. Table 2 contains information about the percentage of participants in each group who fell above, below, and within one standard deviation of the mean.

On the Word Identification test, the mean standard score is 100 and accordingly a score of 85 would fall one standard deviation below the mean. Only one participant in the RRD group scored below 85 on this measure. All other participants in both groups scored within one standard deviation of the mean. These results indicate that reading individual words was not an area of significant difficulty for any of this study's participants.

The mean standard score for the Blending Non-words test is 10 and scores between 8 and 12 fall within one standard deviation of the mean. Almost half of the participants in the RRD group had scores that fell more than one standard deviation below the mean while the majority of the participants in the NRD group scored within one standard deviation of the mean or more than one standard deviation above the mean. Interestingly, however, the results for this measure were not entirely consistent. Three participants in the NRD group scored more than one standard deviation below the mean indicating difficulty with phonological processing and one participant in the RRD group received a standard score of 13 which is more than one standard deviation above the mean and indicates very good phonological processing ability.

On the Nelson-Denny Reading Test, the raw scores for rate and timed comprehension were converted to percentile ranks using the first-year 
McGonnell, Parrila \& Deacon

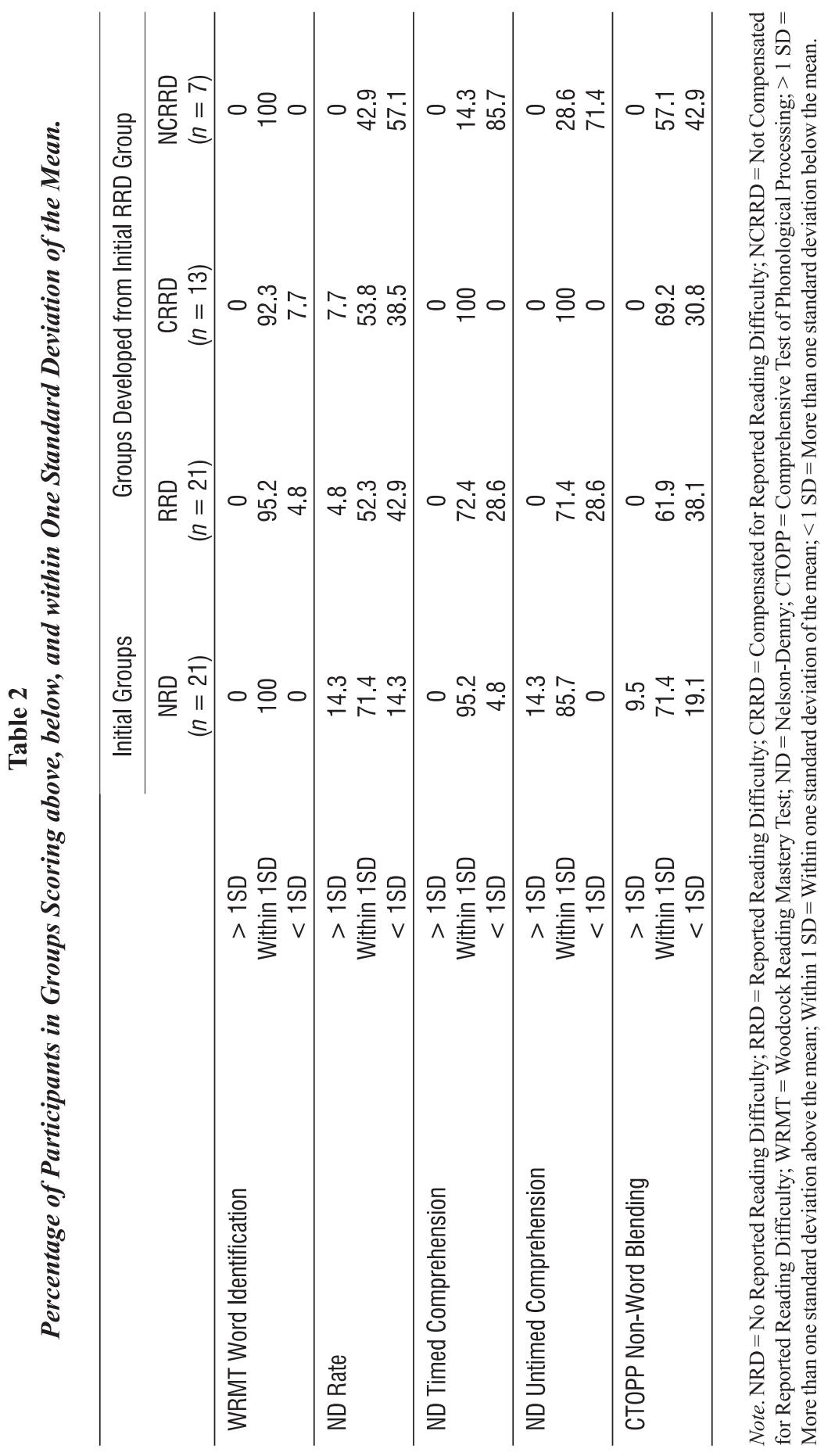


university norms. Here the mean score is at the $50^{\text {th }}$ percentile and scores ranging from the $16^{\text {th }}$ to the $84^{\text {th }}$ percentile fall within one standard deviation of the mean. Six participants in the RRD group and one participant in the NRD group had reading rate scores that fell more than one standard deviation below the mean indicating that they read quite slowly. In terms of timed comprehension, six participants in the RRD group obtained scores that fell more than one standard deviation below the mean. Participants' performance on the measures of reading rate and timed reading comprehension was not always the same. Four of the RRD participants scored more than one standard deviation below the mean on the measures of reading rate and timed reading comprehension. Two participants scored within one standard deviation of the mean on the measure of rate but more than one standard deviation below the mean on the timed measure of reading comprehension. Two other participants scored within one standard deviation of the mean on the timed measure of reading comprehension but more than one standard deviation below the mean on the measure of rate.

In terms of untimed reading comprehension, some participants in both groups exhibited difficulty, but this was the case for twice as many participants in the RRD group as in the NRD group. It is interesting to note that only 9 of the 21 participants in the RRD group correctly answered $80 \%$ or more of the comprehension questions they attempted while 17 of the 21 participants in the NRD group were able to achieve this level of comprehension.

Profiles of RRD Participants across the Tasks. An examination of the results from the individual measures reported above indicates some variability in reading skills of the participants in the RRD group. Eight of the twenty-one participants who reported early difficulty with the acquisition of reading skills scored within or above one standard deviation of the mean on all measures. Four other participants scored within or above one standard deviation of the mean on four of five measures. The exception was phonological processing where these four participants scored more than one standard deviation below the mean. Thus, 12 out of the 21 participants reporting early reading difficulties now performed within the normal range in all direct reading tasks used in this study.

One participant scored more than one standard deviation below the mean on phonological awareness and word identification; however, this participant's word identification score was 84 , only one point away from being within one standard deviation of the mean. This participant may be best placed with the four who only have remaining difficulty with phonological processing. 
Three participants continued to have difficulty with reading rate as demonstrated by lower scores (more than one standard deviation below the mean) on the reading rate and timed reading comprehension measures but good scores $(72 \%, 86 \%, 95 \%)$ on untimed reading comprehension. Four participants continued to demonstrate difficulty with both phonological processing as well as with reading rate and/or timed reading comprehension. One participant demonstrated ongoing difficulty with reading rate and both timed and untimed reading comprehension. This participant scored at the $11^{\text {th }}$ percentile (more than one standard deviation below the mean) on the timed measure of reading comprehension and was only able to answer $44 \%$ of the attempted comprehension questions correctly. This score was more than $20 \%$ below the next lowest untimed reading comprehension score for the RRD participants.

'Compensated' and 'Not Compensated' RRD Participants. Determining which participants in the RRD group can be considered to be 'compensated' requires attention to performance across all reading measures. Clearly, the eight participants who demonstrated no detectible difficulty with any of the tasks can be considered to have compensated for the early difficulties they reported. It also seems reasonable to conclude that the 5 participants who only demonstrated difficulty with phonological processing but not with word identification, reading rate, or reading comprehension can be considered to have compensated for early reported reading difficulty. (This includes the one participant who demonstrated marginal difficulty with word identification.) Therefore, it seems that 13 of the participants who reported early difficulty with the acquisition of reading skills can now be considered to have Compensated for Reported Reading Difficulties (CRRD group).

Seven participants demonstrated difficulty with more than one reading skill including phonological awareness, reading rate, and/or timed reading comprehension. Because these participants continue to have difficulty with many aspects of reading, they can be considered Not Compensated for Reported Reading Difficulties (NCRRD group). The one participant who only demonstrated difficulty with reading comprehension represents a potentially interesting subset of adults with reading difficulties; however, this participant can not be categorized as either Compensated or Not Compensated and will not be included in further analyses. Complete descriptive statistics for the three groups are available in Table 3.

Distinguishing Reading Skills of the CCRD and NRD Groups. Next, we examined whether the measured reading skills for the CRRD group were statistically different from those who did not report any early difficulty. The groups compared in this analysis were the original NRD group $(n=21)$ and the CRRD 


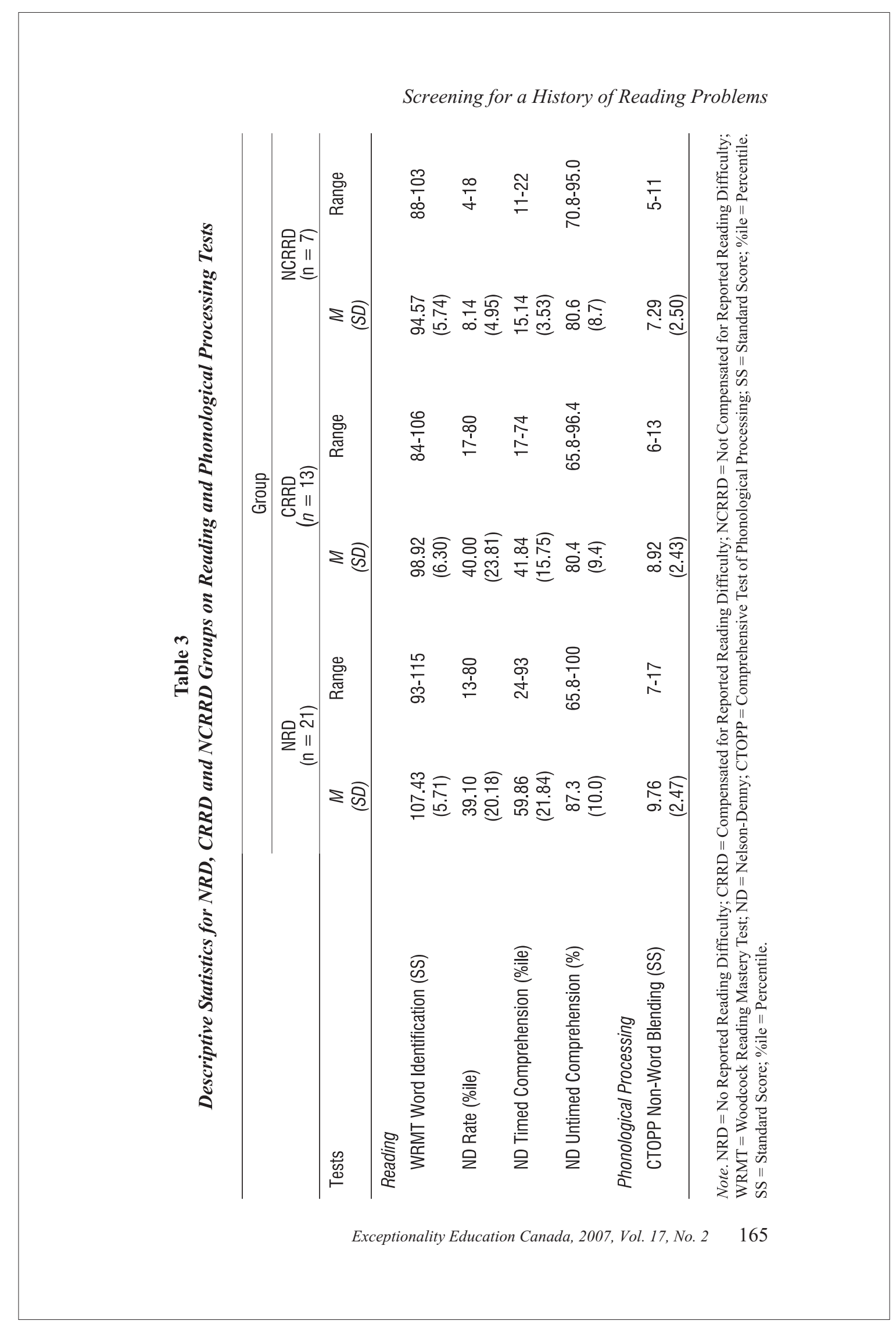


group $(n=13)$. A MANOVA was computed to test for overall group differences. The same five reading skills as in the earlier analyses (word identification, reading rate, timed reading comprehension, untimed reading comprehension, and phonological awareness) were examined. Overall group differences were found, $F(5,28)=3.851, p=.009, \eta^{2}=.405$. The groups differed in terms of word identification, $F(1,32)=14.739, p=.001, \eta^{2}=.315$, $d=1.28$, and timed reading comprehension, $F(1,32)=6.78, p=.014, \eta^{2}=$ $.175, d=.95$, with strong effect sizes for both analyses. There was a trend to significance and a moderate effect size for untimed reading comprehension, $F$ $(1,32)=4.033, p=.053, \eta^{2}=.112, d=.71$. The groups did not differ in terms of either phonological awareness, $F(1,32)=.938, p=.340, \eta^{2}=.028, d=.34$, or reading rate, $F(1,32)=.085, p=.773, \eta^{2}=.003, d=.10$. The null findings for these last two analyses are supported by the respectively small and trivial effect sizes, despite small sample sizes.

\section{Reports of Formal Diagnoses}

The final question investigated by this study was what proportion of university students who report early difficulty with the acquisition of reading skills would also report receiving formal diagnoses of reading disability/dyslexia in childhood or as adults. All participants were asked whether they had ever had any formal or informal assessment and/or diagnosis of reading difficulty.

Eleven participants in the original RRD group (52.4\%) indicated that they had received some form of diagnosis in the past, but only 3 participants (14.3\%) reported receiving a formal diagnosis from a psychologist. These participants were diagnosed with dyslexia (one participant) or a learning disability (two participants). The participant who reported the diagnosis of dyslexia currently demonstrated difficulty only in the area of phonological processing and one of the participants who reported being diagnosed with a learning disability currently demonstrated no detectible reading difficulty. Both and were characterized as being Compensated (CRRD group). The other participant who reported having been diagnosed with a learning disability demonstrated current difficulty with both timed and untimed reading comprehension.

The other 8 participants (38.1\%) noted no formal diagnoses but did report some informal acknowledgement of reading difficulty. They described themselves as having been told they were 'slow' readers, as having needed extra help, or as receiving resource support or program adaptations. Three of these individuals currently demonstrated no detectible difficulty with any reading 
skills and one individual demonstrated specific difficulty with phonological awareness but not with other reading skills. These four participants were characterized as Compensated (CRRD). Four individuals demonstrated current difficulty with more than one reading skill and were characterized as Not Compensated (NCRRD).

Of the 10 students $(47.6 \%)$ who reported no past formal or informal assessment or diagnosis, 5 demonstrated no currently detectible difficulty with any reading skills assessed and 2 with only phonological awareness. These participants were characterized as Compensated (CRRD). The 3 other students demonstrated specific difficulty with reading rate and were characterized as Not Compensated (NCRRD).

\section{Discussion}

The analyses reported here were designed to characterize the current reading skills of university students who self-reported difficulty with the acquisition of these skills. Specifically, the first and most critical question was whether university students who report early difficulty with the acquisition of reading skills (RRD group) demonstrate poorer reading skills than students who do not report early difficulty (NRD group). Overall, the RRD group demonstrated less well developed reading skills than the NRD group. The RRD group had poorer word identification skills and poorer timed and untimed reading comprehension skills than the NRD group. As such, self-report appears to have the ability to distinguish adults who currently have difficulties with reading from those who do not.

There were several findings that indicated the importance of addressing our second question: Do all individuals who report early difficulty with the acquisition of reading skills continue to demonstrated difficulties, or have some managed to compensate for their initial difficulties so they are indistinguishable from those who report no early difficulty? One indication of the importance of this question was the fact that the NRD and RRD groups were not different in all reading skills assessed. Despite indications of trends towards significance, the groups showed statistically similar phonological awareness skills and reading rates. Another indication of the importance of this question was the fact that within the RRD group, scores on the reading measures ranged from significantly below the normal level to what could be considered the high average range. An examination of the reading skills of the individuals within each group led to the conclusion that there were two fairly distinct groups within the RRD 
group, a Compensated (CRRD) and a Not Compensated (NCRRD) group. The CRRD group was designated as compensated because their reading skills (with the exception of phonological awareness for 5 participants) all fell within one standard deviation of the mean standardized score. The NCRRD individuals demonstrated more pervasive difficulty with reading skills (i.e., scores more than one standard deviation below the standardized mean). These individuals had difficulty with reading rate, phonological awareness, and/or reading comprehension. It is important to note that in reality, all members of the RRD group may be compensating for at least some of the early difficulty they report with reading because they were all attending university at the time of their participation in the study. These individuals seem to have developed reading skills or coping strategies that were sufficient to allow them to complete high school with relatively high marks and to manage in some way the volume of reading required by university courses.

The finding that some individuals have compensated to a large extent for early difficulty with reading is consistent with other research (e.g., Lefly \& Pennington, 1991). Approximately 62\% (13 of 21) of the individuals in the current study who reported difficulty with the early acquisition of reading skills had compensated to the point where their reading skills were virtually indistinguishable from those who did not report such early difficulty. This is a much larger proportion than the 22 to $25 \%$ predicted by Lefly and Pennington (1991). It is possible that the cut-off score of .37 in the questionnaire was too liberal for the current population and by itself this led to identifying participants with only minor difficulties with reading acquisition; however, we think that it is reasonable to expect a higher proportion of compensation in the participants in our study for two reasons.

Firstly, the participants in this study were all university students. It is reasonable to expect that those who compensated more effectively for early difficulties with reading would be more likely to attend university than those who have not compensated. This self-selection process could account for the higher proportion of compensated individuals in this study. The overall rate of $62 \%$ in this study is also similar to the approximately $57 \%$ of adult dyslexic participants in Fink's (1998) study who were indistinguishable from a nondyslexic control group on all reading skills assessed. Fink's participants were highly successful university graduates so it is reasonable to expect a similar rate of compensation in the university student population in our study. Secondly, very few participants in this study received a specific diagnosis in childhood. It is possible that our participants' reading difficulties were less severe to begin with and it was therefore easier for them to compensate for their difficulties. 
We were further interested in whether those individuals who had compensated for early reading difficulties would read well enough to be indistinguishable from those who did not report any early difficulty. To answer this question, we compared the reading skills of those in the original NRD group to the CRRD group. Clearly, we need to be concerned with low sample sizes in considering the results of these analyses and yet in the results of the analyses that follow, we will see that there are several significant results and that null results are accompanied by low effect sizes. Consequently, low power does not seem to eliminate the detection of group differences in this study. We turn to analyses of each reading measure in turn.

Overall, the NRD group performed better than the CRRD on the word identification measure despite the fact that 33 of 34 participants performed in what might be considered the 'Average' range (i.e., within one standard deviation of the standardized mean) and that weakness in word identification skill was not a criterion used to create the groups. However, weakness in decoding skills is the hallmark of a reading disability and Deacon et al. (2006) also noted a similar pattern in a population of university students with self-reported early difficulty with reading. (See also Parrila et al. in this issue.) Interestingly, the groups did not differ in terms of phonological awareness which is generally accepted as the core deficit in reading disabilities (Bradley \& Bryant, 1983; Morris et al., 1998; Stanovich \& Siegel, 1994). This could be an indication that the CRRD group has definitely compensated for early difficulty with reading acquisition and now have good phonological awareness skills. Parrila et al. (this issue) also discuss the possibility that some adult readers may have developed a visual method of compensating for their weak phonological awareness skills. It is also possible, however, that those individuals in the CRRD group represent a group for whom phonological awareness was not the core deficit or that early difficulties with phonological awareness received remedial attention and were successfully addressed.

The NRD and CRRD groups did not differ on the measure of reading rate used in this study indicating that the CRRD group could decode as quickly as the NRD group. At first glance, this could be taken to indicate that the CRRD had compensated to the point where they were fluent readers. This does not, however, appear to be the case as the CRRD group performed significantly less well than the NRD group on the timed measure of reading comprehension. These results seem to indicate that the CRRD group continues to have difficulty with integrating the processes involved in reading. While they can read at the same rate as the NRD group, the CRRD group are not able to process the text as efficiently (also indicated by their lower score on word identification) and therefore they extract less meaning in the time they do take to read. It is also 
possible that the CRRD group needs to reread the text to glean its full meaning and that the Nelson-Denny reading rate measure that focuses on the first minute of reading is not able to capture a true reading rate for this group. The fact that the groups were not significantly different on the measure of untimed reading comprehension supports the notion that the CRRD group is able to understand text as well as the NRD group if given sufficient time to compensate for ongoing difficulty with fluency.

The final question addressed by this study was what proportion of university students who report early difficulty with the acquisition of reading skills also reported receiving formal diagnoses of reading disability/dyslexia in childhood or as adults. Only 3 participants in the RRD group reported receiving a formal diagnosis in childhood while no participants reported receiving any formal diagnosis as an adult. Receiving formal or informal acknowledgement of difficulty with reading did not appear to be related to whether participants compensated for early difficulty with reading as 6 of the 11 of those who reported a formal diagnosis or informal acknowledgement and 7 of the 10 individuals who did not report any such acknowledgement or diagnosis were characterized as compensated. More importantly, 8 individuals, only one of whom had a formal diagnosis were still having considerable difficulty with reading skills despite the fact that they were attending university. These individuals must be compensating in some way for these challenges. Discovering how this population manages the reading requirements of a university course-load should be the focus of future research. Further, the existence of this group speaks to the importance of including university participants without formal diagnoses in reading research.

Previous research with adults with reading difficulties (e.g., Deacon et al., 2006) has used self-report in addition to formal documentation of reading difficulty. The incorporation of screening as a methodology has the potential to be extremely useful in studies of adult reading. It is relatively inexpensive and efficient for large-scale use and screening by using self-report has the potential to produce a more ecologically valid sample by including participants who have not received formal diagnoses. Traditional procedures used to recruit adults for research into reading difficulties can result in the exclusion of these individuals. However, it will be important for researchers choosing to adopt this screening method to be aware that those who self-report early difficulty with reading appear to present with variable reading skills as adults.

It will also be important to consider that self-report relies on remembered perception of early reading difficulty and that the perception of difficulty does not mean that an actual difficulty existed. A perception can of course arise from 
a variety of sources. An individual may have had extremely gifted siblings to whom he or she was constantly compared or may have encountered a teacher whose instructional style did not match his/her learning needs. Because school is such a large part of children's lives and reading such a large part of school, negative experiences that might have had little to do with an individual's reading skills can leave a strong, emotionally laden impression that reading was difficult. Consequently, it is possible that research using self-report as the only screening measure will include individuals with and without actual disabilities.

It should be noted that this study did not assess the spelling skills of the participants. This limitation should be addressed in future research. Fink (1998) found that a sub-group of her adult participants had compensated for early reading difficulties in all areas except spelling ability. Additional information about spelling ability could provide further evidence for the existence of 'compensated' and 'not compensated' individuals in a university population. It is also possible that some individuals who appear to have compensated for early difficulty with reading acquisition will continue to show difficulty specifically in the area of spelling and/or written expression skills. This also has implications for the ability of adults to be successful in university and in life.

University students who have managed to compensate to varying degrees for early reading difficulties have the potential to be a rich source of information about pathways to compensation that could benefit children by pointing to more effective methods of remediation. As such, this population should continue to be the focus of research and this study would indicate that using self-report as a screening measure can be effective for recruitment. However, the presence of individuals who demonstrate significant difficulty with many reading skills in a university population has other, less positive, implications as well. It seems that some of the students in this study, particularly those who have not compensated for early reading difficulty, will likely have difficulties coping with the demands of university classes. None of these individuals in our study had any formal diagnosis and it would therefore be difficult for them to obtain what could be appropriate and necessary accommodations through student services. Finally, only half of the participants in the RRD group had even an informal indication that they had difficulty with reading and only 3 had received any formal diagnosis of reading difficulty. These facts point to challenges within the educational system. All these individuals were aware that reading was somewhat problematic for them, but few received acknowledgement of or assistance with these difficulties. This gap could be addressed by implementing a broad assessment of the reading skills of all children which could provide the basis for longitudinal work to examine the pathways children follow to compensate for early reading difficulty. 
McGonnell, Parrila \& Deacon

\section{References}

Birch, S., \& Chase, C. (2004). Visual and language processing deficits in compensated and uncompensated college students. Journal of Learning Disabilities, 37, 389-410.

Bradley, L., \& Bryant, P. E. (1983). Categorising sounds and learning to read-a causal connection. Nature, 301, 419-420.

Brown, J. I., Fishco, V. V., \& Hanna, G. (1993). The Nelson-Denny Reading Test. Itasca, IL: Riverside Publishing Company.

Bruck, M. (1990). Word-recognition skills of adults with childhood diagnoses of dyslexia. Developmental Psychology, 24, 439-454.

Bruck, M. (1992). Persistence of dyslexics' phonological awareness deficits. Developmental Psychology, 28, 874-886.

Deacon, S. H., Parrila, R., \& Kirby, J. R. (2006). Processing of derived forms in high-functioning dyslexics. Annals of Dyslexia, 56, 103-128.

Decker, S. N., Vogler, G. P., \& Defries, J. C. (1989). Validity of self-reported reading disability by parents of reading-disabled control children. Reading and Writing: An Interdisciplinary Journal, 1, 327-331.

Erskine, J. M., \& Seymour, P. H. K. (2005). Proximal analysis of developmental in adulthood: The cognitive mosaic model. Journal of Educational Psychology, 97, 406-424.

Fink, R. P. (1998). Literacy development in successful men and women with dyslexia. Annals of Dyslexia, 48, 311-346.

Lefly, D. L. \& Pennington, B. F. (1991). Spelling errors and reading fluency in compensated dyslexics. Annals of Dyslexia, 41, 143-162.

Lefly, D. L. \& Pennington, B. F. (2000). Reliability and validity of the Adult Reading History Questionnaire. Journal of Learning Disabilities, 33, 286-296.

Leong, C. K. (1999). Phonological and morphological processing in adult students with learning/reading disabilities. Journal of Learning Disabilities, 32, 224-238.

Miller-Shaul, S. (2005). The characteristics of young and adult dyslexic readers on reading and reading related cognitive tasks as compared to normal readers. Dyslexia, 11, 132-151. 
Morris, R. D., Stuebing, K. K., Fletcher, J. M., Shaywitz, S. E., Lyon, G. R., Shankweiler, D. P., et al. (1998). Subtypes of reading disability: Variability around a phonological core. Journal of Educational Psychology, 90, 347-373.

Parrila, R., Corkett, J., Kirby, J. R. \& Hein, S. (2003). Adult Reading History Questionnaire - Revised. Unpublished questionnaire. University of Alberta.

Schulte-Körne, G., Deimel, W., \& Remschmidt, H. (1997). Can self-report data on deficits in reading and spelling predict spelling disability as defined by psychometric tests? Reading and Writing: An Interdisciplinary Journal, 9, 55-63.

Stanovich, K. E., \& Siegel, L. S. (1994). Phenotypic performance profile of children with reading disabilities: A regression- based test of phonological-core variable-difference model. Journal of Educational Psychology, $86,24-53$

Wagner, R. K., Torgesen, J. K., \& Rashotte, C. A. (1999). The Comprehensive Test of Phonological Processing. Austin, TX: PRO-ED.

Wilson, A. M., \& Lesaux, N. K. (2001). Persistence of phonological processing deficits in college students with dyslexia who have age-appropriate reading skills. Journal of Learning Disabilities, 34, 394-400.

Woodcock, R. W. (1987). Woodcock Reading Mastery Tests-Revised. Circle Pines, MN: American Guidance Service.

\section{Authors' Note}

Correspondence concerning this article should be addressed to:

Melissa McGonnell, Department of Psychology, LSC, Dalhousie University, 1355 Oxford Street, Halifax, NS, B3H 4J1. E-mail: melissa.mcgonnell@dal.ca 
McGonnell, Parrila \& Deacon

174 Exceptionality Education Canada, 2007, Vol. 17, No. 2 